\title{
Sparse finite elements for elliptic problems with stochastic loading
}

\section{Journal Article}

Author(s):

Schwab, Christoph; Todor, Radu Alexandru

Publication date:

2003-10

Permanent link:

https://doi.org/10.3929/ethz-b-000053177

Rights / license:

In Copyright - Non-Commercial Use Permitted

Originally published in:

Numerische Mathematik 95(4), https://doi.org/10.1007/s00211-003-0455-z 


\section{Sparse finite elements for elliptic problems with stochastic loading ${ }^{\star}$}

\section{Christoph Schwab, Radu-Alexandru Todor}

Seminar for Applied Mathematics, ETH-Zentrum, 8092 Zürich, Switzerland; e-mail: schwab@math.ethz.ch

Received September 5, 2001 / Revised version received October 31, 2002 /

Published online April 17, 2003 - (C) Springer-Verlag 2003

Summary. We formulate elliptic boundary value problems with stochastic loading in a bounded domain $D \subset \mathbb{R}^{d}$. We show well-posedness of the problem in stochastic Sobolev spaces and we derive a deterministic elliptic PDE in $D \times D$ for the spatial correlation of the random solution. We show wellposedness and regularity results for this PDE in a scale of weighted Sobolev spaces with mixed highest order derivatives. Discretization with sparse tensor products of any hierarchic finite element (FE) spaces in $D$ yields optimal asymptotic rates of convergence for the spatial correlation even in the presence of singularities or for spatially completely uncorrelated data. Multilevel preconditioning in $D \times D$ allows iterative solution of the discrete equation for the correlation kernel in essentially the same complexity as the solution of the mean field equation.

Mathematics Subject Classification (2000): 65N30

\section{Introduction}

Due to the rapid development of scientific computing in recent years, accurate numerical solution of boundary value problems for partial differential equations is now possible in many applications. For given problem data, such as domains, coefficients and boundary data, the solution can be computed to high accuracy. Often, however, the problem data is either incompletely known

Correspondence to: C. Schwab

* Research performed under IHP network Breaking Complexity of the EC, contract number HPRN-CT-2002-00286, and supported in part by the Swiss Federal Office for Science and Education under grant number BBW 02.0418. 
or uncertain which implies that highly accurate numerical solutions are of limited use. One way to deal with such uncertainty is to describe the problem data as random fields which turns the problem into a stochastic differential equation. The formulation and numerical solution of stochastic differential equations has received increasing interest in recent years. We mention here only [18], [19], [16] and the references there on stochastic ordinary differential equations and [12], [13], [7] on stochastic partial differential equations. In engineering simulations, uncertainty in coefficients and loadings has been dealt with by means of the stochastic finite element method in structural mechanics (see [15] and the references there) and by the related first order, second moment perturbation technique in subsurface flow models, introduced in [6].

The solution of a stochastic differential equation is, in general, a random field which takes values in a suitable function space. Complete description of this random field requires knowledge of its joint probability densities. In applications, however, one is often only interested in the first moments of the random solution. These moments can be computed e.g. by the Monte-Carlo (MC) Method, where numerous 'samples' of the random input data are generated according to prescribed, often empirical, distributions and each MC sample entails the solution of a deterministic boundary value problem. From the computed solutions, the mean and covariance then give estimates for the first moments of the random solution. This approach is costly - due to the generally slow convergence of MC methods, numerous samples must be taken until a satisfactory accuracy of the computed solution has been reached. Nevertheless, in the context of stochastic ordinary differential equations, this technique is frequently employed (e.g. [16]) with good success. For partial differential equations, one could discretize in the spatial variables first, e.g. by the Finite Element Method (FEM). This will then lead to large linear systems with random stiffness and mass matrices, the so-called stochastic FEM [15]. The cost of this approach is often prohibitive, particularly in 3-d.

Alternatively one can directly compute the moments of interest for the random solution and this is the approach which we follow here. This approach consists in deriving deterministic partial differential equations for the moments of the random solution, thereby eliminating the need for MC simulations. This advantage is bought, however, at a price: if the differential equation is posed in the physical domain $D \subset \mathbb{R}^{d}$, the 2 nd moment of the solution, the spatial correlation, is a function in $D \times D \subset \mathbb{R}^{2 d}$. We show in the present paper for elliptic partial differential equations with stochastic input data that the deterministic equation for the correlation has a very special structure. We exploit this structure for anisotropic regularity estimates which in turn show that finite element approximations of the correlation can be computed in essentially the same complexity as FE solutions of the deterministic problem in $D$ by sparse tensor products of FE spaces. 
We now specify the problems to be considered. Let $(\Omega, \Sigma, P)$ be a $\sigma$-finite probability space and $D \subset \mathbb{R}^{d}$ a bounded open set with Lipschitz boundary $\partial D$. Let $A \in L^{\infty}\left(D, \mathbb{R}_{\text {sym }}^{d \times d}\right)$ be also positive definite, i.e. for some $\alpha, \beta>0$ there holds

$$
\alpha\|\xi\|^{2} \leq \xi^{\top} A(x) \xi \leq \beta\|\xi\|^{2} \quad \forall \xi \in \mathbb{R}^{d} \text { and } \lambda \text { - a.e. } x \in D .
$$

We define a random field on a submanifold $M$ of $\mathbb{R}^{d}$ (it will be always $D$ or some part of its boundary) as a jointly measurable function from $M \times \Omega$ to $\mathbb{R}$. Suppose $\partial D=\Gamma_{0} \cup \Gamma_{1}$ is a disjoint union of closed subsets, where $\Gamma_{0}$ has positive surface measure and let $f, g$ and $h$ be random fields on $D, \Gamma_{0}$ and $\Gamma_{1}$ respectively. We consider the following model problem, with stochastic r.h.s.,

$$
\left.\begin{array}{r}
L\left(\partial_{x}\right) u \\
\gamma_{0}(u) \\
\gamma_{n}(u)
\end{array}\right\}:=\left\{\begin{array}{r}
-\operatorname{div}(A(x) \nabla u(x, \omega)) \\
\left.u(x, \omega)\right|_{\Gamma_{0}} \\
\left.n^{\top} A(x) \nabla u(x, \omega)\right|_{\Gamma_{1}}
\end{array}\right\}= \begin{cases}f(x, \omega) & \text { in } D \\
g(x, \omega) & \text { on } \Gamma_{0}, \\
h(x, \omega) & \text { on } \Gamma_{1}\end{cases}
$$

where the operators involved in the boundary conditions should be thought of as stochastic counterparts of the classical trace on $\Gamma_{0}$ or $\Gamma_{1}$ and distributional conormal derivative operators, $\gamma_{0}, \gamma_{1}$ and $\gamma_{n}$ respectively.

We mention that in practice, one is also interested in solving a problem with stochastic coefficient $A(x, \omega)$, where usually $A(x, \omega)$ is an $\epsilon$-small perturbation of its mean field w.r.t. $\omega$. The Keller method [14] represents the stochastic solution as an exponentially convergent series, in which each term solves a stochastic problem with the same deterministic coefficient but different stochastic loadings. Clearly then, a rigorous study of (1.2), which is the purpose of this work, is a key step towards efficient numerical methods for the more general case of a stochastic coefficient.

The present paper is organized as follows. In Section 2, we first introduce appropriate function spaces of data $(f, g, h)$ and solutions $u$ in such a way that (1.2) becomes a well-posed problem. In Section 3 we define the 'statistics of $u$ ', and we derive deterministic partial differential equations which describe them. The 'statistics of $u$ ' that we are interested in here are the moments of first and second order of the random solution $u(x, \omega)$ to (1.2), sometimes referred to as the mean field (or expectation) $E_{u}$ and correlation $C_{u}$ of $u(x, \omega)$. They are defined by

$$
E_{u}(x):=\int_{\Omega} u(x, \omega) d P(\omega), \quad C_{u}(x, y):=\int_{\Omega} u(x, \omega) u(y, \omega) d P(\omega),
$$

whenever these quantities exist. Section 4 addresses the regularity of the correlation equation, in particular also in polygonal domains. Section 5 discusses the finite element approximation of the correlation equation. We describe a sparse FE space which allows to achieve, in terms of the number of degrees 
of freedom, the same convergence rates as for the mean field problem in the domain $D$. Section 6 addresses the preconditioning and the iterative solution of the linear system of equations for the correlation problem. Also, a detailed complexity analysis is given and logarithmic linear complexity of the algorithm is established. Finally, Section 7 presents numerical experiments which confirm the theoretical estimates.

\section{Preliminaries}

\subsection{Stochastic Sobolev spaces}

The most appropriate tools for the study of (1.2) are the stochastic Sobolev spaces, which we shall define as tensor products of usual function spaces. Within the setting of the previous section, we consider $L^{2}(\Omega, d P)$, the Hilbert space of all real random variables on $\Omega$ with finite second moments, equipped with the usual inner product

$$
\langle u, v\rangle:=\int_{\Omega} u(\omega) v(\omega) d P(\omega) .
$$

Our convention will be that whenever $H$ is a Sobolev space, $\mathcal{H}$ will denote its stochastic counterpart, that is, the tensor product of $H$ and $L^{2}(\Omega, d P)$. For instance, we shall use

$$
\begin{gathered}
\mathcal{H}^{k}(D):=H^{k}(D) \otimes L^{2}(\Omega, d P), \quad \mathcal{L}^{2}(D)^{d}:=L^{2}(D)^{d} \otimes L^{2}(\Omega, d P) \\
\mathcal{H}^{1 / 2}\left(\Gamma_{0}\right):=H^{1 / 2}\left(\Gamma_{0}\right) \otimes L^{2}(\Omega, d P), \quad \text { etc. }
\end{gathered}
$$

The stochastic Sobolev spaces are equipped with natural Hilbert structures induced from the tensor product factors. Embedding and trace theorems similar to the usual ones hold also true on account of the fact that bounded linear operators between Hilbert spaces can be tensorised. Hence we shall use the following operators,

$$
\begin{aligned}
\nabla & \in \mathcal{B}\left(H^{1}(D), L^{2}(D)^{d}\right) & \nabla \otimes \operatorname{Id} & \in \mathcal{B}\left(\mathcal{H}^{1}(D), \mathcal{L}^{2}(D)^{d}\right) \\
\operatorname{div} & \in \mathcal{B}\left(L^{2}(D) d, H^{-1}(D)\right) & \operatorname{div} \otimes \operatorname{Id} & \in \mathcal{B}\left(\mathcal{L}^{2}(D)^{d}, \mathcal{H}^{-1}(D)\right) \\
\gamma_{j} & \in \mathcal{B}\left(H^{1}(D), H^{1 / 2}\left(\Gamma_{j}\right)\right) & \gamma_{j} \otimes \operatorname{Id} & \in \mathcal{B}\left(\mathcal{H}^{1}(D), \mathcal{H}^{1 / 2}\left(\Gamma_{j}\right)\right), \quad j=0,1
\end{aligned}
$$

Further notations will be

$$
\begin{aligned}
& H_{(0)}^{1}(D):=\operatorname{Ker} \gamma_{0}=\left\{u \in H^{1}(D) \mid \gamma_{0} u=0\right\} \\
& \mathcal{H}_{(0)}^{1}(D):=\operatorname{Ker}\left(\gamma_{0} \otimes \mathrm{Id}\right) \\
& \mathcal{H}^{-1}(D):=\left(H_{(0)}^{1}(D) \otimes L^{2}(\Omega, d P)\right)^{*} \simeq H^{-1}(D) \otimes L^{2}(\Omega, d P),
\end{aligned}
$$

where $\mathcal{B}$ denotes the space of bounded linear operators between two Hilbert spaces, and we have used the usual notation $\mathcal{K}^{\star}$ for the dual space of a Hilbert space $\mathcal{K}$. We always identify $L^{2}(D)$ with its dual, via the Riesz isomorphism. 
As for the coefficient $A(x),(1.1)$ ensures the positivity of the associated multiplication operator, that is, for all $u \in \mathcal{L}^{2}(D)^{d}$ it holds

$$
\alpha\|u\|_{\mathcal{L}^{2}(D)^{d}}^{2} \leq\langle(A \otimes \mathrm{Id}) u, u\rangle_{\mathcal{L}^{2}(D)^{d}} \leq \beta\|u\|_{\mathcal{L}^{2}(D)^{d}}^{2} .
$$

Regarding the stochastic Sobolev spaces, we remark that the norm on $\mathcal{H}_{(0)}^{1}(D)$ is given by

$$
\|\cdot\|_{1}:=\|(\nabla \otimes \mathrm{Id}) \cdot\|_{\mathcal{L}^{2}(D)^{d}},
$$

since the scalar product in $\mathcal{H}_{(0)}^{1}(D)$ is obtained by tensorizing the scalar products $\langle\nabla \cdot, \nabla \cdot\rangle_{L^{2}(D)^{d}}$ in $H_{(0)}^{1}(D)$ and (2.1) in $L^{2}(\Omega, d P)$.

The following result justifies the terminology 'random fields' for the elements of the tensor product spaces introduced above (see, e. g. [23]).

Proposition 1 We have the canonical isomorphisms

$$
H^{k}\left(D ; L^{2}(\Omega, d P)\right) \simeq \mathcal{H}^{k}(D) \simeq L^{2}\left(\Omega, d P ; H^{k}(D)\right) .
$$

\subsection{Random solutions}

We give next a variational formulation of problem (1.2).

Proposition 2 Assume that $f \in \mathcal{H}^{-1}(D), g \in \mathcal{H}^{1 / 2}\left(\Gamma_{0}\right)$ and $h \in \mathcal{H}^{-1 / 2}\left(\Gamma_{1}\right)$. Then there exists a unique random solution $u \in \mathcal{H}^{1}(D)$ such that $\left(\gamma_{0} \otimes \mathrm{Id}\right) u=$ $g$ and

$$
\begin{aligned}
& \langle(A \otimes \mathrm{Id})(\nabla \otimes \mathrm{Id}) u,(\nabla \otimes \mathrm{Id}) v\rangle_{\mathcal{L}^{2}(D)^{d}} \\
& \quad=\langle f, v\rangle_{\mathcal{H}^{-1}(D), \mathcal{H}_{(0)}^{1}(D)}+\left\langle h,\left(\gamma_{1} \otimes \mathrm{Id}\right) v\right\rangle_{\mathcal{H}^{-1 / 2}\left(\Gamma_{1}\right), \mathcal{H}^{1 / 2}\left(\Gamma_{1}\right)}
\end{aligned}
$$

for all $v \in \mathcal{H}_{(0)}^{1}(D)$.

Proof. Since $H^{1}(D) / H_{(0)}^{1}(D) \simeq H^{1 / 2}\left(\Gamma_{0}\right)$ as topological spaces, there exists $u_{1} \in \mathcal{H}^{1}(D)$ such that $\left(\gamma_{0} \otimes \mathrm{Id}\right)\left(u_{1}\right)=g$. The result follows then from the Lax-Milgram Lemma applied in $\mathcal{H}_{(0)}^{1}(D)$, since on account of (2.1) and (2.2), the bilinear form defined by the l.h.s. of (2.5) is bounded and coercive on $\mathcal{H}_{(0)}^{1}(D)$, while the r.h.s. of the problem for $u-u_{1}$ defines a bounded linear functional on the same space.

Remark 1 If we choose $(e)_{i \geq 1}$ to be an ONB in $L^{2}(\Omega ; d P)$ and if we expand $f=\sum_{i} f_{i} \otimes e_{i}$ with $\sum_{i}\left\|f_{i}\right\|_{L^{2}(D)}^{2} \leq \infty$, as well as $g$ and $h$ accordingly, then the solution of (1.2) can be written as a series $u=\sum_{i} u_{i} \otimes e_{i}$ which 
converges absolutely in $\mathcal{H}^{1}(D)$ and whose coefficient functions $u_{i}$ solve the deterministic mixed boundary value problems

$$
P\left(u_{i}\right):=\left\{\begin{array}{r}
L\left(\partial_{x}\right) u_{i} \\
\gamma_{0}\left(u_{i}\right) \\
\gamma_{n}\left(u_{i}\right)
\end{array}\right\}=\left\{\begin{array}{l}
f_{i} \text { in } D \\
g_{i} \text { on } \Gamma_{0} \\
h_{i} \text { on } \Gamma_{1}
\end{array}\right\}, \quad i=1,2, \ldots
$$

This can be seen by choosing the test function in (2.5) of the form $v=w \otimes e_{i}$, with $w \in H_{(0)}^{1}(D)$. Note that the deterministic character of $A$ is essential in this decomposition.

\section{Statistics of $\mathbf{u}$}

In this section we obtain deterministic equations for the expectation and the correlation of the random solution $u$. While the expectation $E_{u}(x)$ of the random solution $u(x, \omega)$ at $x \in D$ is obviously of interest, its spatial correlation $C_{u}\left(x, x^{\prime}\right)$ allows, for instance, to obtain the variance of the random solution $u(x, \omega)$ at $x \in D$ via

$$
\operatorname{Var}(u(x, \cdot))=C_{u}(x, x)-\left(E_{u}(x)\right)^{2}, \quad x \in D .
$$

\subsection{Second order moments}

We shall first give the definition of the correlation of a pair $(u, v)$ when $u, v \in \mathcal{H}^{1}(D)$ and we shall then introduce the expectation of $u$ as the correlation of the pair $(u, 1)$ where $1 \in \mathcal{H}^{1}(D)$ is the tensor product of constant functions equal to 1 on $D$ and $\Omega$ respectively. It is easy to prove that

Proposition 3 Let $u$ and $v$ be elements of $\mathcal{H}^{1}(D)$ and let $\left(e_{i}\right)_{i \geq 1}$ be an $O N B$ in $L^{2}(\Omega ; d P)$, so that $u=\sum_{i} u_{i} \otimes e_{i}$, where $u_{i} \in H^{1}(D) \forall i \geq 1$ and $\sum_{i}\left\|u_{i}\right\|_{H^{1}(D)}^{2}<\infty$. Define $v_{i}$ similarly. Then $\sum_{i} u_{i} \otimes v_{i}$ converges in $H^{1}(D) \otimes H^{1}(D)$ and the limit does not depend on the choice of the basis $\left(e_{i}\right)_{i \geq 1}$.

The previous result motivates the following

Definition 1 If $u$ and $v$ are elements of $\mathcal{H}^{1}(D)$, then the series $C_{u, v}$ defined in Proposition 3 is called the correlation of the pair $(u, v)$. If $u=v$ we write $C_{u}$ instead of $C_{u, u}$ and speak about the correlation of $u$.

Remark 2 From the proof it follows also that if $H, H_{1}, H_{2}$ are separable Hilbert spaces, and $u \in H_{1} \otimes H, v \in H_{2} \otimes H$, the correlation $C_{u, v}$ can be defined as an element of $H_{1} \otimes H_{2}$. We shall use this for $H_{1}=H^{-1}(D)$ and $H_{2}=L^{2}(S ; d m)$ where $(S, \Upsilon, m)$ is a $\sigma$-finite measure space, or for $H_{1}=H_{2}=H^{-1}(D)$. In this way one can construct the correlations of the pairs $(f, h)$ and $(f, f)$ with $f$ and $h$ as in Proposition 2. 


\subsection{Equation for $C_{u}$}

We define the expectation, or mean field, of $u$ by $E_{u}:=C_{u, 1}=\int_{\Omega} u(x, \omega)$ $d P(\omega)$. The expectation $E_{u}$ of the random solution $u(x, \omega)$ satisfies a deterministic boundary value problem which is easily derived. We choose an ONB $\left(e_{i}\right)_{i \geq 1}$ in $L^{2}(\Omega ; d P)$ with $e_{1}=1$ (the constant function equal to 1 on $\Omega$ ), so that $E_{u}=u_{1}$ is the unique solution of a mixed boundary value problem with data $f_{1}=C_{f, 1}=: E_{f}, g_{1}=C_{g, 1}=: E_{g}, h_{1}=C_{h, 1}=: E_{h}$, as follows from Remark 1

$$
P\left(E_{u}\right)=\left\{\begin{array}{c}
L\left(\partial_{x}\right) E_{u} \\
\gamma_{0}\left(E_{u}\right) \\
\gamma_{n}\left(E_{u}\right)
\end{array}\right\}=\left\{\begin{array}{c}
E_{f} \text { in } D, \\
E_{g} \text { on } \Gamma_{0}, \\
E_{h} \text { on } \Gamma_{1} .
\end{array}\right\} .
$$

For future reference, we recall here also the variational formulation.

Find $E_{u} \in\left\{E_{g}\right\}+H_{(0)}^{1}(D)$ such that

$$
q\left(E_{u}, v\right)=l(v) \quad \forall v \in H_{(0)}^{1}(D),
$$

where

$$
\begin{aligned}
q(u, v) & :=\langle A \nabla u, \nabla v\rangle_{L^{2}(D)^{d}}, \\
l(v) & :=\left\langle E_{f}, v\right\rangle_{H^{-1}(D), H_{(0)}^{1}(D)}+\left\langle E_{h}, v\right\rangle_{L^{2}\left(\Gamma_{1}\right)} .
\end{aligned}
$$

To give a weak deterministic equation for the correlation function, we introduce, following [1], anisotropic Sobolev spaces on $D \times D$ by

$$
\begin{aligned}
& H^{k, l}(D \times D):=H^{k}(D) \otimes H^{l}(D) \\
& H_{(0)}^{k, l}(D \times D):=H_{(0)}^{k}(D) \otimes H_{(0)}^{l}(D)
\end{aligned}
$$

for all integers $k, l \geq 1$. Using also the notation $L^{2}(D \times D)^{d \times d}$ for $L^{2}(D)^{d} \otimes$ $L^{2}(D)^{d}$, we consider the following operators acting on the anisotropic spaces,

$$
\begin{aligned}
& \nabla_{x, y}:=\nabla_{x} \otimes \nabla_{y} \quad \in \mathcal{B}\left(H^{1,1}(D \times D), L^{2}(D \times D)^{d \times d}\right) \\
& \gamma_{j, x, y}:=\gamma_{j . x} \otimes \gamma_{j, y} \in \mathcal{B}\left(H^{1,1}(D \times D), L^{2}\left(\Gamma_{j} \times \Gamma_{j}\right)\right) \text { for } j=0,1 \\
& A_{x, y}:=A_{x} \otimes A_{y} \in \mathcal{B}\left(L^{2}(D \times D)^{d \times d}\right) .
\end{aligned}
$$

The correlation of $u$ given by (2.5) satisfies a fourth-order elliptic equation in $D \times D$ and that the bilinear form involved is coercive (see also [5]). As it can be easily seen, if $u$ solves (2.5), then $C_{u}$ satifies also the following boundary conditions

$$
\left(\gamma_{0} \otimes \mathrm{Id}\right) C_{u}=C_{g, u} \text { and }\left(\mathrm{Id} \otimes \gamma_{0}\right) C_{u}=C_{u, g}
$$

on $\Gamma_{0} \times D$ and $D \times \Gamma_{0}$ respectively. We shall assume homogeneous Dirichlet boundary condition on $\Gamma_{0}, g=0$. In view of the fact that the trace operator of $D \times D$ on $\left(\Gamma_{0} \times \bar{D}\right) \cup\left(\bar{D} \times \Gamma_{0}\right)$ is $\left(\gamma_{0} \otimes \mathrm{Id}\right) \oplus\left(\mathrm{Id} \otimes \gamma_{0}\right)$, (3.3) means, if $g=0$, that $C_{u} \in H_{(0)}^{1,1}(D \times D)$. Moreover, it holds 
Proposition 4 Assume that $u$ is the solution of (2.5) with $h \in \mathcal{L}^{2}\left(\Gamma_{1}\right), f \in$ $\mathcal{L}^{2}(D)$ and $g=0$. Then the correlation $C_{u}$ of the random solution $u(x, \omega)$ is the unique solution in $H_{(0)}^{1,1}(D \times D)$ of

$$
C_{u} \in H_{(0)}^{1,1}(D \times D): \quad \mathcal{Q}\left(C_{u}, C\right)=\mathcal{L}(C) \quad \forall C \in H_{(0)}^{1,1}(D \times D),
$$

where the bilinear form $\mathcal{Q}$ is given by

$$
\mathcal{Q}\left(C_{u}, C\right):=\left\langle A_{x, y} \nabla_{x, y} C_{u}, \nabla_{x, y} C\right\rangle_{L^{2}(D \times D)^{d \times d}}
$$

and the load functional is

$$
\begin{aligned}
\mathcal{L}(C):= & \left\langle C_{f}, C\right\rangle_{L^{2}(D \times D)}+\left\langle C_{h, f},\left(\gamma_{1} \otimes \mathrm{Id}\right) C\right\rangle_{L^{2}\left(\Gamma_{1} \times D\right)} \\
& +\left\langle C_{f, h},\left(\mathrm{Id} \otimes \gamma_{1}\right) C\right\rangle_{L^{2}\left(D \times \Gamma_{1}\right)}+\left\langle C_{h},\left(\gamma_{1} \otimes \gamma_{1}\right) C\right\rangle_{L^{2}\left(\Gamma_{1} \times \Gamma_{1}\right)}
\end{aligned}
$$

Proof. Expand $C=\sum_{i} w_{i} \otimes v_{i}$ where $\left(v_{i}\right)_{i \geq 1}$ is an ONB in $H_{(0)}^{1}(D)$ and $\left(w_{i}\right)_{i \geq 1} \subset H_{(0)}^{1}(D)$ with $\sum_{i}\left\|w_{i}\right\|_{H_{(0)}^{1}(D)}^{2}<\infty$. Then

$$
\begin{aligned}
\mathcal{Q}\left(C_{u}, C\right)= & \sum_{i, j}\left\langle A_{x} \nabla_{x} u_{i} \otimes A_{y} \nabla_{y} u_{i}, \nabla_{x} w_{j} \otimes \nabla_{y} v_{j}\right\rangle \\
= & \sum_{i, j}\left\langle A_{x} \nabla_{x} u_{i}, \nabla_{x} w_{j}\right\rangle\left\langle A_{y} \nabla_{y} u_{i}, \nabla_{y} v_{j}\right\rangle \\
= & \left\langle\sum_{i} f_{i} \otimes f_{i}, \sum_{j} w_{j} \otimes v_{j}\right\rangle \\
& +\left\langle\sum_{i} h_{i} \otimes f_{i}, \sum_{j} \gamma_{1} w_{j} \otimes v_{j}\right\rangle \\
& +\left\langle\sum_{i} f_{i} \otimes h_{i}, \sum_{j} w_{j} \otimes \gamma_{1} v_{j}\right\rangle \\
& +\left\langle\sum_{i} h_{i} \otimes h_{i}, \sum_{j} \gamma_{1} w_{j} \otimes \gamma_{1} v_{j}\right\rangle
\end{aligned}
$$

where all scalar products are $L^{2}$ on appropriate domains and all series converge absolutely. As (3.6) defines a continuous linear functional on $H_{(0)}^{1,1}(D \times$ $D$ ), we have to check, in order to ensure the uniqueness of a solution for (3.4), only the boundedness and coercivity in the same space of the sesquilinear form (3.5). But this follows at once from the boundedness and the strict positivity of the operator $A_{x, y}$ in $L^{2}(D \times D)^{d \times d}$ (with lower and upper bounds $\alpha^{2}$ and $\beta^{2}$ respectively), and from the fact that $\|\cdot\|_{1}:=\left\|\nabla_{x, y} \cdot\right\|_{L^{2}(D \times D) d \times d}$ is a norm on $H_{(0)}^{1,1}(D \times D)$.

Remark 3 The proof of Proposition 4 can be easily modified in order to cover also the more general situation $f \in \mathcal{H}^{-1}(D)$. Note that in this case one should replace in the definition (3.6) of the functional $\mathcal{L}$ the $L^{2}(D)$ scalar product by the duality pairing $H_{(0)}^{1}(D) \times H^{-1}(D)$. 
Remark 4 If $C_{f, h} \neq E_{f} \otimes E_{h}, f$ and $h$ are said to be correlated. As it is readily seen, the superposition principle does not hold for (3.4) due to the non-linearity of the correlations $C_{f}, C_{f, h}, C_{h, f}$.

\section{Regularity}

\subsection{Shift theorem}

Here we derive a regularity result for the weak solution of the correlation equation (3.4). As we shall see, this follows from elliptic regularity, applied in a suitable fashion. We therefore collect first standard results for the mean field problem (3.2).

Definition 2 The problem (3.2) admits a shift theorem at order $s \geq 0$ if $E_{f} \in$ $H^{s-1}(D), E_{g} \in H^{s+1 / 2}\left(\Gamma_{0}\right)$ and $E_{h} \in H^{s-1 / 2}\left(\Gamma_{1}\right)$ imply $E_{u} \in H^{s+1}(D)$ and the dependence of the solution on data is continuous in these spaces.

Sufficient conditions for a shift theorem at order $s \geq 0$ are given e.g. in [8].

Proposition 5 Assume that $\partial D \in C^{\infty}$, and that the entries of $A$ are of class $C^{s, 1}(\bar{D})$ with $s>0$. Then the problem (3.2) admits a shift theorem at order $s$.

A shift theorem for the mean field equation (3.2) carries over to the correlation problem (3.4), as follows. We assume, again for convenience, $g=0$.

Proposition 6 Suppose that (3.2) satisfies the shift theorem at order s. Then the correlation problem (3.4) admits a shift theorem at order $s$ in spaces of mixed highest derivatives. More precisely, if

$$
\begin{aligned}
& C_{f} \in H^{s-1, s-1}(D \times D), \quad C_{h} \in H^{s-1 / 2, s-1 / 2}\left(\Gamma_{1} \times \Gamma_{1}\right) \\
& C_{f, h} \in H^{s-1, s-1 / 2}\left(D \times \Gamma_{1}\right), C_{h, f} \in H^{s-1 / 2, s-1}\left(\Gamma_{1} \times D\right),
\end{aligned}
$$

then $C_{u} \in H^{s+1, s+1}(D)$.

Proof. In the case $g=0$ the operator $P^{-1}$ which associates to each element of $H^{s-1}(D) \oplus H^{s-1 / 2}\left(\Gamma_{1}\right)$ the corresponding solution of the problem (3.2) is a homeomorphism on $H^{s+1}(D) \cap H_{(0)}^{1}(D)$. We deduce that $P^{-1} \otimes P^{-1}$ is a homeomorphism from $H:=\left(H^{s-1}(D) \otimes H^{s-1}(D)\right) \oplus\left(H^{s-1}(D) \otimes\right.$ $\left.H^{s-1 / 2}\left(\Gamma_{1}\right)\right) \oplus\left(H^{s-1 / 2}\left(\Gamma_{1}\right) \otimes H^{s-1}(D)\right) \oplus\left(H^{s-1 / 2}\left(\Gamma_{1}\right) \otimes H^{s-1 / 2}\left(\Gamma_{1}\right)\right)$ onto its range in $H^{s+1, s+1}(D) \cap H_{(0)}^{1,1}(D \times D)$. We still have to check that $P^{-1} \otimes P^{-1}$ sends the quadruple $\left(C_{f}, C_{f, h}, C_{h, f}, C_{h}\right)$ into the solution $C_{u}$ of the corresponding problem (3.4). It is enough to prove this for $\left(f_{1}, h_{1}\right) \otimes$ $\left(f_{2}, h_{2}\right)$, in view of the density of the span of such elements in $H$. To this end, we note that $\left(P^{-1} \otimes P^{-1}\right)\left(\left(f_{1}, h_{1}\right) \otimes\left(f_{2}, h_{2}\right)\right)=u_{1} \otimes u_{2}$, where $u_{1}$ and $u_{2}$ solve the classical boundary value problem (2.6) with data $\left(f_{1}, 0, h_{1}\right)$ and $\left(f_{2}, 0, h_{2}\right)$ respectively. Upon multiplying the variational formulations of these two problems we obtain the desired conclusion. 


\subsection{Nonsmooth domains}

If $\partial D$ is not smooth, the problem (3.2) admits a shift theorem at order $s$ only for $0 \leq s<s^{*}$ with a small $s^{*}>0$ (depending on the smoothness of $\partial D$ and $A$ ). In such situations we also have a shift theorem at order $s \geq s^{*}$ in weighted Sobolev spaces. We exemplify this in dimension $d=2$. Let $D \subset \mathbb{R}^{2}$ be a bounded polygon with $M$ vertices $A_{i}, i=1, \ldots, M$ and straight sides $\Gamma_{i}, i=1, \ldots, M$ connecting $A_{i}$ and $A_{i+1}$ (we set $A_{M+1}=A_{1}$ ). Denote by $\omega_{i}$ the size of the interior angle at vertex $A_{i}$. For $x \in D, r_{i}(x)$ is the distance from $x$ to $A_{i}$ and we associate with each $A_{i}$ an exponent $\beta_{i} \in(0,1)$. We write $\beta=\left(\beta_{1}, \beta_{2}, \ldots, \beta_{M}\right)$ and, for $k \in \mathbb{Z}, \beta+k:=\left(\beta_{1}+k, \beta_{2}+k, \ldots, \beta_{M}+k\right)$.

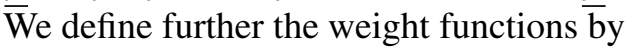

$$
\omega_{\underline{\beta}+k}(x):=\prod_{i=1}^{M}\left(r_{i}(x)\right)^{\beta_{i}+k} .
$$

The weighted Sobolev spaces $H_{\beta}^{k, l}(D)$ are defined as closures of $C^{\infty}(\bar{D})$ under the norms

$$
\|u\|_{H_{\beta}^{k, l}(D)}^{2}:=\|u\|_{H^{l-1}(D)}^{2}+\sum_{|\underline{\alpha}|=l}^{k}\left\|\omega_{\underline{\beta}+|\alpha|-l} \mathrm{D}^{\alpha} u\right\|_{L^{2}(D)}^{2},
$$

if $k \geq l \geq 0$. Then it holds (see e.g. [2])

Proposition 7 Assume that $D \subset \mathbb{R}^{2}$ is a polygon with $M$ straight sides and that $A_{i j}(x) \in C^{\infty}(\bar{D})$. Assume further that the boundary data $E_{g}, E_{h}$ in (3.2) admit liftings $E_{G} \in H_{\beta}^{s+1,2}(D), E_{H} \in H_{\beta}^{s, 1}(D)$ for some $s \geq 0$. Then there exist numbers $\beta_{i} \in[0,1), i=1, \ldots, M$ such that for any $k \in \mathbb{N}_{0}$ and $E_{f} \in H_{\beta}^{k, 0}(D)$ the solution $E_{u}$ of (3.2) belongs to $H_{\beta}^{k+2,2}(D)$. Moreover, denoting $s:=k+1$, there holds a shift theorem at order $s$ in weighted spaces,

$$
\left\|E_{u}\right\|_{H_{\beta}^{s+1,2}(D)} \lesssim\left\|E_{f}\right\|_{H_{\beta}^{s-1,0}(D)}+\left\|E_{G}\right\|_{H_{\beta}^{s+1,2}(D)}+\left\|E_{H}\right\|_{H_{\beta}^{s, 1}(D)} .
$$

Since solution singularities can only appear on a measure zero subset of $\partial D$ (i.e. at vertices), a trace operator $\operatorname{Tr}$ on $\Gamma_{1}$ can be defined as an $L^{0}\left(\Gamma_{1}\right)$-valued linear operator on $H_{\beta}^{s, 1}(D)$, where by $L^{0}\left(\Gamma_{1}\right)$ we denote the space of measurable functions on $\Gamma_{1}$. It is trivial to see that the kernel of this trace operator is closed in $H_{\beta}^{s, 1}(D)$. This enables us to define further $H_{\beta}^{s-1 / 2,1 / 2}\left(\Gamma_{1}\right):=$ $H_{\beta}^{s, 1}(D) / \operatorname{Ker}(\operatorname{Tr})$ as a Banach-space, with the usual inf-norm. Passing in (4.4) to the infimum over all $H \in H_{\beta}^{s, 1}(D)$ with the same trace $h$, we obtain that the operator which associates to each pair $(f, h)$ the solution $u$ of (3.2) with $g=0$ is a homeomorphism from $H_{\beta}^{s-1,0}(D) \otimes H_{\beta}^{s-1 / 2,1 / 2}\left(\Gamma_{1}\right)$ to $H_{\beta}^{s+1,2}(D) \cap H_{(0)}^{1}(D)$. In view of the fact that a tensor product of linear 
homeomorphisms between Hilbert spaces is again a homeomorphism, we obtain, using the same argument as in Proposition 6, the following regularity result.

Proposition 8 Assume that $D \subset \mathbb{R}^{2}$ is a polygon with straight sides and that the problem (3.2) admits a shift estimate (4.4) at order $s \geq 0$ in weighted spaces. Assume further that the data are sufficently regular, namely that for some positive $s \in \mathbb{R}$ holds

$$
\begin{aligned}
& C_{f, f} \in H_{\beta}^{s-1,0}(D) \otimes H_{\beta}^{s-1,0}(D), C_{h, h} \in H_{\beta}^{s-1 / 2,1 / 2}\left(\Gamma_{1}\right) \otimes H_{\beta}^{s-1 / 2,1 / 2}\left(\Gamma_{1}\right) \\
& C_{f, h} \in H_{\beta}^{s-1,0}(D) \otimes H_{\beta}^{s-1 / 2,1 / 2}\left(\Gamma_{1}\right), C_{h, f} \in H_{\beta}^{s-1 / 2,1 / 2}\left(\Gamma_{1}\right) \otimes H_{\beta}^{s-1,0}(D) .
\end{aligned}
$$

Then

$$
C_{u} \in H_{\beta}^{s+1,2}(D) \otimes H_{\beta}^{s+1,2}(D) .
$$

We apply the basic regularity result Proposition 6 to two frequently used examples of spatial correlation functions.

\subsection{Exponential correlation}

We consider a second order process $f$ with correlation function

$$
C_{f}(x, y)=e^{-c|x-y|}, \quad(x, y) \in D \times D,
$$

where $c>0$ is a parameter and the bounded domain $D \subset \mathbb{R}^{d}$ is smooth. Note that this correlation kernel can be used to characterize the well-known Markovian processes. For various examples of such processes we refer to [22].

To deduce the regularity of $C_{f}$ given by (4.5), we use the following two auxiliary results (see also [20]).

Lemma 1 Let $u: \mathbb{R}^{d} \rightarrow \mathbb{R}$ be defined by $u(x)=\exp (-|x|)$. Then $u \in$ $H^{s}\left(\mathbb{R}^{d}\right)$, for $s<d / 2+1$.

Lemma 2 If $s=p+q$ with $p, q \geq 0$ and $f \in H^{s}\left(\mathbb{R}^{d}\right)$, then the function $u: \mathbb{R}^{d} \times \mathbb{R}^{d} \rightarrow \mathbb{R}$ defined by

$$
u(x, y):=f(x-y) \quad \text { a.e. }(x, y) \in \mathbb{R}^{d} \times \mathbb{R}^{d}
$$

belongs to $H_{\mathrm{loc}}^{p, q}\left(\mathbb{R}^{d} \times \mathbb{R}^{d}\right)$.

Proof. We have to show that if $\phi, \psi \in C_{0}^{\infty}\left(\mathbb{R}^{d}\right)$, the function $v: \mathbb{R}^{d} \times \mathbb{R}^{d} \rightarrow$ $\mathbb{R}$ defined a.e. by $v(x, y):=\phi(x) \psi(y) f(x-y)$ belongs to $H^{p, q}\left(\mathbb{R}^{d} \times \mathbb{R}^{d}\right)$. We remark that it suffices to show that the function $w: \mathbb{R}^{d} \times \mathbb{R}^{d} \rightarrow \mathbb{R}$ defined a.e. by $w(x, y):=\psi(y) f(x-y)$ belongs to $H^{p, q}\left(\mathbb{R}^{d} \times \mathbb{R}^{d}\right)$, since the multiplication operator by $\phi$ is bounded in $H^{p}\left(\mathbb{R}^{d}\right)$ and tensorizing it 
by the identity of $H^{q}\left(\mathbb{R}^{d}\right)$ produces again a bounded operator, this time in $H^{p, q}\left(\mathbb{R}^{d} \times \mathbb{R}^{d}\right)$. In view of the fact that the Fourier transform and tensor product commute, all we have to check is $\left(\langle\xi\rangle:=\left(1+|\xi|^{2}\right)^{1 / 2}\right)$

$$
\langle\xi\rangle^{p}\langle\eta\rangle^{q} \hat{w}(\xi, \eta) \in L_{\xi, \eta}^{2}\left(\mathbb{R}^{d} \times \mathbb{R}^{d}\right) .
$$

Explicit computation of the Fourier transform of $w$ in terms of those of $f$ and $\psi$ shows that

$$
\hat{w}(\xi, \eta)=\hat{\psi}(\xi+\eta) \hat{f}(\xi) .
$$

Using (4.8), (4.7) can be then written

$$
\frac{\langle\xi\rangle^{p}\langle\eta\rangle^{q}}{\langle\xi+\eta\rangle^{q}\langle\xi\rangle^{s}} \cdot\langle\xi+\eta\rangle^{q} \hat{\psi}(\xi+\eta) \cdot\langle\xi\rangle^{s} \hat{f}(\xi) \in L_{\xi, \eta}^{2}\left(\mathbb{R}^{d} \times \mathbb{R}^{d}\right) .
$$

But this follows if we note that, by assumption on $f$, the last of the three factors in (4.9) belongs to $L_{\xi}^{2}\left(\mathbb{R}^{d}\right)$, the second one belongs to $L_{\eta}^{2}\left(\mathbb{R}^{d}\right)$, uniformly in $\xi$ $\left(\hat{\psi} \in \mathcal{S}\left(\mathbb{R}^{d}\right)\right.$ ), while the first is bounded uniformly in $\xi$ and $\eta$, since $s=p+q$ and the inequality $\sqrt{2}\langle x\rangle\langle y\rangle\rangle\langle x+y\rangle$ holds for all $x, y \in \mathbb{R}^{d}$.

As a direct consequence of the previous two lemmas and the boundedness of the restriction operator from $H_{\mathrm{loc}}^{s}\left(\mathbb{R}^{d}\right)$ to $H^{s}(D)$ for all $s \geq 0, d \in \mathbb{N}^{*}$, the correlation kernel (4.5) satisfies

$$
C_{f}(x, y)=e^{-c|x-y|} \in H^{(d+2) / 4-\epsilon,(d+2) / 4-\epsilon}(D \times D), \quad \forall \epsilon>0 .
$$

We can therefore apply Proposition 6 with $\Gamma_{1}=\emptyset$, and deduce

Proposition 9 If (3.2) admits a shift theorem at level $s \geq 0$ in $H^{s+1}(D)$ and if the correlation of the data $f$ is $C_{f}(x, y)=e^{-c|x-y|}$ for some $c>0$, then the solution $C_{u}$ of (3.4) with $g=0$ and $\Gamma_{1}=\emptyset$ belongs, for any $\epsilon>0$, to $H^{t, t}(D \times D)$, where

$$
t=\min ((d+10) / 4-\epsilon, s+1),
$$

and

$$
\left\|C_{u}\right\|_{H^{t, t}(D \times D)} \lesssim\left\|C_{f}\right\|_{H^{t-2, t-2}(D \times D)},
$$

with a constant depending only on $t$ and $d$.

This result shows that the regularity of $C_{u}$ in a polygon $D \subset \mathbb{R}^{2}$, measured in $H^{s}(D)$, with $C_{f}=e^{-c|x-y|}$, is determined by corner singularities, since $s<2$ for a reentrant corner. 


\subsection{Vanishing spatial correlation}

Here we consider that $D$ is a bounded Lipschitz domain in $\mathbb{R}^{d}$, with $d \leq 3$ and $\Gamma_{1}=\emptyset$ which ensures $H_{(0)}^{1,1}(D \times D)=H_{0}^{1,1}(D \times D)$. We denote further by $\Delta_{D}$ the diagonal set of $D \times D$, and we consider also an arbitrary function $k \in L^{2}\left(\Delta_{D}\right)$. We let then $k \cdot \delta(x-y)$ be the distribution defined by

$$
\langle k \cdot \delta(x-y), \phi\rangle=\int_{\Delta_{D}} k(x) \phi(x, x) d x \quad \forall \phi \in C_{0}^{\infty}(D \times D) .
$$

Note that one can view the correlation kernel $\delta(x-y)$ as a limiting case of exponential-type correlations described in the previous section, due to

$$
c^{d}\left(\int_{\mathbb{R}^{d}} e^{-|z|} d z\right)^{-1} e^{-c|x-y|} \stackrel{c \rightarrow \infty}{\longrightarrow} \delta(x-y) \text { in } \mathcal{D}^{\prime}\left(\mathbb{R}^{d} \times \mathbb{R}^{d}\right)
$$

Vanishing spatial correlations lead formally to the problem of finding $C_{u} \in$ $H_{(0)}^{1,1}(D \times D)$ such that

$$
\mathcal{Q}\left(C_{u}, C_{v}\right)=\left\langle k \cdot \delta(x-y), C_{v}\right\rangle \quad \forall C_{v} \in H_{(0)}^{1,1}(D \times D) .
$$

The solvability of (4.12) depends on the admissibility of the data (4.11), and this follows for $d \leq 3$ from

Lemma 3 ([21]) If $D$ is a bounded Lipschitz domain in $\mathbb{R}^{d}$, the trace operator $R: C^{\infty}(\bar{D} \times \bar{D}) \longrightarrow L^{2}\left(\Delta_{D}\right)$,

$$
R(\phi)(x)=\phi(x, x) \quad \forall x \in D
$$

has a unique linear continuous extension from $H^{s, s}(D \times D)$ to $L^{2}\left(\Delta_{D}\right)$, provided that $s>d / 4$.

Lemma 3 and Proposition 6 imply

Proposition 10 If a shift theorem at level $s \geq 0$ holds for problem (3.2) in $D \subset \mathbb{R}^{d}, d \leq 3$, then there exists a unique weak solution $C_{u}$ solution of (4.12) and it belongs, for any $\epsilon>0$, to $H^{t, t}(D \times D)$, where

$$
t=\min (2-d / 4-\epsilon, s+1) .
$$

Moreover, the following a-priori estimate holds, with a constant depending only on $t$ and $d$,

$$
\left\|C_{u}\right\|_{H^{t, t}(D \times D)} \lesssim\|k\|_{L^{2}(\Delta)} \cdot\|R\|_{\mathcal{B}\left(H^{2-t, 2-t}(D \times D), L^{2}(\Delta)\right)} .
$$




\section{Discretization}

\subsection{FE spaces and approximation properties}

We investigate the Finite Element approximation of the statistics of $u$. Let $\left\{V^{L}\right\}_{L \geq 0}$ be a dense, hierarchical sequence of finite dimensional subspaces of $H_{(0)}^{1}(D)$, that is,

$$
V^{0} \subset V^{1} \subset \ldots \subset V^{L} \subset \ldots \subset H_{(0)}^{1}(D),
$$

where $N^{L}=\operatorname{dim}\left(V^{L}\right)<\infty$ for all $L$ (here $L$ stands for the level). We assume also that the following approximation property holds,

$$
\min _{v \in V^{L}}\|u-v\|_{H_{(0)}^{1}(D)} \leq \Phi\left(N^{L}, s\right)\|u\|_{H^{s+1}(D)},
$$

for all $u \in H^{s+1}(D) \cap H_{(0)}^{1}(D)$, with $\Phi(N, s) \rightarrow 0$ for $s>0$ as $N \rightarrow \infty$. For regular solutions the usual $\mathrm{FE}$ spaces based on quasiuniform, shape regular meshes are suitable.

Example 1 Let $\left\{\mathcal{T}^{L}\right\}_{L \in \mathbb{N}}$ be a nested sequence of regular, simplicial triangulations of the domain $D$ of meshwidth $h^{L}=h^{L-1} / 2, \forall L \geq 1$ and let $p \geq 1$ be a polynomial degree. Then

$$
V^{L}:=S^{p}\left(D, \mathcal{T}^{L}\right):=\left\{u \in C^{0}(\bar{D}):\left.u\right|_{K} \in \mathcal{P}^{p}(K) \quad \forall K \in \mathcal{T}^{L}\right\}
$$

satisfies (5.1), as well as (5.2), with $\Phi(N, s)=O\left(N^{-\delta}\right)$, for fixed $p$ and $L \rightarrow \infty$, where $\delta:=\min \{p, s\} / d$.

Remark 5 If $D \subset \mathbb{R}^{2}$ is a polygon, problem (3.2) admits a shift theorem at order $s$ in the spaces $H^{s+1}(D)$ only for small values of $s$ (often $1 / 2<s<1$ ). In this case, however, for smooth data in (3.2), we still have a shift theorem at order $s \geq 0$ in the weighted spaces $H_{\beta}^{1+s, 2}(D)$ with some $\underline{\beta} \in(0,1)^{M}$, i.e. the weight function $\omega_{\underline{\underline{\beta}}+k}(x)$ introduced in Section 4.1 compensates for the corner singularities of the solution $u$. To the weighted spaces $H_{\beta}^{k, l}(D)$ correspond FE approximations on sequences of graded meshes $\left\{\mathcal{T}_{\gamma}^{n}\right\}_{n}^{-}$with shape-regular elements which satisfy in dimension 2

$$
\begin{gathered}
\left|\mathcal{T}_{\gamma}^{n}\right|:=\# \text { of triangles in } \mathcal{T}_{\gamma}^{n}=O\left(n^{2}\right), \\
\forall T \in \mathcal{T}_{\gamma}^{n}: h_{T}:=\operatorname{diam}(T) \leq C \omega(x)^{1-1 / \gamma_{n}} n^{-1},
\end{gathered}
$$

for $\gamma \geq 1$. Clearly, $\gamma=1$ corresponds to quasiuniform triangulations of meshwidth $h=O\left(n^{-1}\right)$, whereas $\gamma \gg 1$ corresponds to strong refinement near the vertices. Then, for any $u \in H_{\underline{\beta}}^{1+s, 2}(D)$ we have, as $n \rightarrow \infty$

$$
\inf _{v \in S^{p}\left(D, \mathcal{T}_{\gamma}^{n}\right)}\|u-v\|_{H^{1}(D)} \lesssim N^{-\delta}\|u\|_{H_{\underline{\beta}}^{1+s, 2}(D)},
$$

with $\delta:=\min \{p, s\} / d$, provided that $\gamma>\min \{p, s\} / \operatorname{Re} \lambda$, where $\operatorname{Re} \lambda>0$ denotes the real part of the smallest singularity exponent of the solution $u$ in the polygon $D$. 


\subsection{Rate of convergence for $C_{u}$}

The standard approximation result for the mean field problem (3.2) with the FE spaces of Example 1 reads,

Proposition 11 Assume that the mixed boundary value problem (3.2) for $E_{u}$ satisfies the shift theorem at order $s \geq 0$. Then the FE approximation $E_{u}^{L} \in$ $V^{L}$ of $E_{u}$, the solution of (3.2) with data $E_{f} \in H^{s-1}(D), E_{g} \in H^{s+1 / 2}\left(\Gamma_{0}\right)$, $E_{h} \in H^{s-1 / 2}\left(\Gamma_{1}\right)$, reads

$$
E_{u}^{L} \in S^{p}\left(D, \mathcal{T}^{L}\right), \quad q\left(E_{u}^{L}, E_{v}\right)=l\left(E_{v}\right) \quad \forall E_{v} \in S^{p}\left(D, \mathcal{T}^{L}\right) .
$$

Then, with $\delta=\min \{p, s\} / d$, we have the following error estimate, asymptotically as $N^{L} \rightarrow \infty$,

$$
\left\|E_{u}-E_{u}^{L}\right\|_{H^{1}(D)} \lesssim\left(N^{L}\right)^{-\delta}\left\|E_{u}\right\|_{H^{s+1}(D)} .
$$

We investigate next the FE approximation of the correlation kernel $C_{u}$ in the deterministic elliptic equation (3.4). Since $C_{u}$ solves an elliptic problem on $D \times D$, we construct $\mathrm{FE}$ spaces in this product domain, starting from $\left\{V^{L}\right\}_{L \geq 0}$ in (5.1). Full tensor product spaces $\left\{V^{L} \otimes V^{L}\right\}_{L \geq 0}$ present themselves as natural candidates. We next prove that, for the regularity (4.1) of $C_{u}$, the sparse tensor product FE spaces, defined by (see [24])

$$
\hat{V}^{L}:=\operatorname{Span}\left\{V^{i} \otimes V^{j} \quad \mid 0 \leq i+j \leq L\right\} \subset H_{(0)}^{1,1}(D \times D)
$$

allow to approximate $C_{u}$ at essentially the same rate. Sparse tensor product spaces can be described in terms of a hierarchic excess of the scale (5.1), that is, of an algebraic summand $W^{L}$ of $V^{L-1}$ in $V^{L}$,

$$
W^{L}:=V^{L} \ominus V^{L-1} \quad L \geq 0,
$$

where we set $V^{-1}:=\{0\}$. It follows that $V^{L}$ decomposes as a direct sum

$$
V^{L}=\bigoplus_{0 \leq i \leq L} W^{i}
$$

while the similar decomposition of the full tensor product $F E$ spaces in $D \times D$ reads

$$
\bar{V}^{L}:=V^{L} \otimes V^{L}=\bigoplus_{0 \leq i, j \leq L} W^{i} \otimes W^{j} \subset H_{(0)}^{1,1}(D \times D),
$$

for all $L \in \mathbb{N}$. The sparse tensor product spaces (5.6) are then given by

$$
\hat{V}^{L}=\bigoplus_{0 \leq i+j \leq L} W^{i} \otimes W^{j} .
$$


For an arbitrary $C_{u} \in H_{(0)}^{1,1}(D \times D)$ we define $C_{u}^{L}$, the sparse interpolant of $C_{u}$ in $\hat{V}^{L}$, as the $H_{(0)}^{1,1}(D \times D)$ projection of $C_{u}$ on $\hat{V}^{L}$. With these notations, the following result (see also [11], [17]) shows that the approximation property of the scale $\left\{V^{L}\right\}_{L \geq 0}$ carries over to the sparse scale $\left\{\hat{V}^{L}\right\}_{L \geq 0}\left(\Phi\left(N_{-1}, s\right)\right.$ is defined as the embedding constant of $H^{1}(D)$ in $\left.H^{s+1}(D)\right)$.

Proposition 12 Assume that the sequence (5.1) of FE spaces $\left\{V_{L}\right\}_{L \geq 0}$ has the approximation property (5.2). Then for $C_{u} \in H_{(0)}^{1,1}(D \times D) \cap H^{s+1, t+1}(D \times$ $D)$ with $s, t>0$ the sparse interpolant $C_{u}^{L}$ approximates $C_{u}$ with the error $e_{u}^{L}:=C_{u}-C_{u}^{L}$,

$$
\begin{aligned}
\left\|e_{u}^{L}\right\|_{H_{(0)}^{1,1}(D \times D)} \leq & {\left[\sum_{i=0}^{L+1} \Phi^{2}\left(N^{i-1}, s\right) \Phi^{2}\left(N^{L-i}, t\right)\right]^{1 / 2}\left\|C_{u}\right\|_{H^{s+1, t+1}(D \times D)} } \\
& +\left[\sum_{i=L+1}^{\infty} \Phi^{2}\left(N^{i}, s\right)\right]^{1 / 2}\left\|C_{u}\right\|_{H^{s+1,1}(D \times D)} .
\end{aligned}
$$

Proof. Let us denote by $P^{L}$ the $H_{(0)}^{1}(D)$ orthogonal projection onto $V^{L}$. We choose $W^{L}$ to be the orthogonal complement of $V^{L-1}$ in $V^{L}(\forall L \geq 0)$ and use (5.10) to orthogonally decompose $C_{u}$ and $C_{u}^{L}$ as

$$
C_{u}=\sum_{0 \leq i, j}\left(Q^{i} \otimes Q^{j}\right) C_{u}, \quad C_{u}^{L}=\sum_{0 \leq i+j \leq L}\left(Q^{i} \otimes Q^{j}\right) C_{u},
$$

where $Q^{i}$ denotes the orthogonal projection $P^{i}-P^{i-1}$ on $W^{i}$. (5.12) implies then, all norms being evaluated in $H_{(0)}^{1,1}(D \times D)$,

$$
\begin{aligned}
\left\|e_{u}^{L}\right\|^{2} & =\left\|\sum_{i+j \geq L+1}\left(Q^{i} \otimes Q^{j}\right) C_{u}\right\|^{2} \\
& =\left\|\sum_{i=0}^{\infty} \sum_{j \geq \max \{L+1-i, 0\}}\left(Q^{i} \otimes Q^{j}\right) C_{u}\right\|^{2} \\
& =\left\|\sum_{i=0}^{L+1} Q^{i} \otimes\left(\operatorname{Id}-P^{L-i}\right) C_{u}\right\|^{2}+\left\|\sum_{i=L+2}^{\infty} Q^{i} \otimes \operatorname{Id} C_{u}\right\|^{2} .
\end{aligned}
$$

Since for all $u \in H_{(0)}^{1}(D) \cap H^{s+1}(D)$ we have

$$
\begin{aligned}
\left\|Q^{i} u\right\|_{H_{(0)}^{1}(D)} & \leq\left\|\left(\mathrm{Id}-P^{i-1}\right) u\right\|_{H_{(0)}^{1}(D)} \\
\left\|\left(\operatorname{Id}-P^{L}\right) u\right\|_{H_{(0)}^{1}(D)} & \leq \Phi\left(N^{L}, s\right)\|u\|_{H^{s+1}(D)},
\end{aligned}
$$

the estimate (5.11) follows by using (5.15) and (5.14) in (5.13). 
Specializing the FE spaces $V^{L}$ as in Example 1, we obtain that the FE approximation $\hat{C}_{u}^{L} \in \hat{V}^{L}$ of $C_{u}$ requires, for a prescribed accuracy, essentially $O\left(N^{L}\right)$ degrees of freedom.

Proposition 13 Assume that the mean field problem (3.1) satisfies the shift theorem at order $s \geq 0$ and that the correlation functions of the data satisfy $C_{f} \in H^{s-1, s-1}(D \times D), C_{f, h} \in H^{s-1, s-1 / 2}\left(D \times \Gamma_{1}\right), C_{h, f} \in$ $H^{s-1 / 2, s-1}\left(\Gamma_{1} \times D\right)$ and $C_{h} \in H^{s-1 / 2, s-1 / 2}\left(\Gamma_{1} \times \Gamma_{1}\right)$.

Then the sparse FE approximation $\hat{C}_{u}^{L}$ of the correlation function $C_{u}$ which is defined by

$$
\hat{C}_{u}^{L} \in \hat{V}^{L}, \quad \mathcal{Q}\left(\hat{C}_{u}^{L}, C\right)=\mathcal{L}(C) \quad \forall C \in \hat{V}^{L},
$$

converges, as $L \rightarrow \infty$, with the rate

$$
\left\|C_{u}-\hat{C}_{u}^{L}\right\|_{H_{(0)}^{1,1}(D \times D)} \lesssim\left(\log N^{L}\right)^{1 / 2}\left(N^{L}\right)^{-\delta}\left\|C_{u}\right\|_{H^{s+1, s+1}(D \times D)}
$$

where $\delta=\min \{p, s\} / d$.

Proof. The coercivity of the sesquilinear form $\mathcal{Q}$ defined in (3.5) has been proved in Proposition 4. A direct consequence of this fact is the quasi-optimality of the FE solution $\hat{C}_{u}^{L}\left(\|\cdot\|\right.$ is here the norm in $\left.H_{(0)}^{1,1}(D \times D)\right)$,

$$
\left\|C_{u}-\hat{C}_{u}^{L}\right\| \lesssim \min _{C \in \hat{V}^{L}}\left\|C_{u}-C\right\|=\left\|C_{u}-C_{u}^{L}\right\| .
$$

with a constant depending only on the bounds $\alpha$ and $\beta$. The quadruple $\left(C_{f}, C_{f, h}, C_{h, f}, C_{h}\right)$ satisfies the regularity assumptions that enable us to apply Proposition 6 and to deduce that $C_{u} \in H^{s+1, s+1}(D \times D)$. Since $N^{j}=$ $O\left(2^{d j}\right),(5.11)$ with $s=t$ shows that

$$
\left\|C_{u}-C_{u}^{L}\right\|_{H_{(0)}^{1,1}(D \times D)} \lesssim\left(\log N^{L}\right)^{1 / 2}\left(N^{L}\right)^{-\delta}\left\|C_{u}\right\|_{H^{s+1, s+1}(D \times D)}
$$

From this and (5.18) follows then the claimed estimate. Note also, for later use, that, due to (5.10), it holds, asymptotically as $L \rightarrow \infty$,

$$
\hat{N}^{L}:=\operatorname{dim}\left(\hat{V}^{L}\right)=O\left(L 2^{d L}\right)=O\left(\left(\log N^{L}\right) N^{L}\right),
$$

which allows us to express the convergence rate (5.17) in terms of the number of degrees of freedom $\hat{N}^{L}$ in $D \times D$,

$$
\left\|C_{u}-\hat{C}_{u}^{L}\right\|_{H_{(0)}^{1,1}(D \times D)} \lesssim\left(\log \hat{N}^{L}\right)^{1 / 2+\delta}\left(\hat{N}^{L}\right)^{-\delta}\left\|C_{u}\right\|_{H^{s+1, s+1}(D \times D)} .
$$


Remark 6 The FE approximation $\bar{C}_{u}^{L}$ of $C_{u}$,

$$
\bar{C}_{u}^{L} \in \bar{V}^{L}, \quad \mathcal{Q}\left(\bar{C}_{u}^{L}, C\right)=\mathcal{L}(C) \quad \forall C \in \bar{V}^{L},
$$

based on the full tensor product space $\bar{V}^{L}$ in (5.9) satisfies, under the regularity assumptions in Proposition 13,

$$
\left\|C_{u}-\bar{C}_{u}^{L}\right\|_{H_{(0)}^{1,1}(D \times D)} \lesssim\left(N^{L}\right)^{-\delta / 2}\left\|C_{u}\right\|_{H^{s+1, s+1}(D \times D)} .
$$

We see that for a given regularity of the data, the rate (5.22) in terms of the number of degrees of freedom is essentially half of (5.17).

Remark 7 If $D$ is nonsmooth, it follows from Proposition 8 and Remark 5 that the influence of corner singularities in $D \times D$ can be compensated by forming sparse tensor-products of FE spaces in $D$ with judicious mesh refinement towards the vertices of $D$. Once good meshes for the solution $E_{u}$ of (3.2) have been determined, the sparse FE space for $C_{u}$ based on these meshes will also give optimal rates of convergence for $\hat{C}_{u}^{L}$, provided $C_{f}, C_{f, h}, C_{h, f}, C_{h}$ in Proposition 8 are sufficiently regular.

\section{Implementation aspects and complexity}

The discretized correlation equation consists in solving a linear system

$$
\hat{A}^{L} \underline{C}_{u}^{L}=\underline{C}_{f}^{L},
$$

where $\hat{A}^{L}$ denotes the stiffness matrix of (5.16) with respect to some basis of the sparse tensor product space $\hat{V}^{L} \subset H_{(0)}^{(1,1)}(D \times D)$. As it is well-known, the conjugate gradient method generates an approximating sequence $\left\{\underline{C}_{u, n}^{L}\right\}_{n \geq 0}$ satisfying

$$
\left\|\underline{C}_{u}^{L}-\underline{C}_{u, n}^{L}\right\|_{2} \leq 2 \cdot\left(\frac{\sqrt{\kappa}-1}{\sqrt{\kappa}+1}\right)^{n}\left\|\underline{C}_{u}^{L}-\underline{C}_{u, 0}^{L}\right\|_{2}, \quad n=0,1,2 \ldots,
$$

where $\kappa=\operatorname{cond}_{2}\left(\hat{A}^{L}\right)$ and $\|\cdot\|_{2}$ denotes the Euclidian norm (see e.g. [9]). The efficiency of this method relies on the well-conditioning (or boundedness of $\kappa$ as $L \rightarrow \infty$ ) and the sparsity of the matrix involved, $\hat{A}^{L}$. We shall see that a wavelet preconditioning procedure can be used to ensure the well-conditioning, while the sparsity, which fails to hold, can be replaced by a systematic use of the tensor product structure of the correlation equation. We derive an algorithm which employs this special structure to perform the multiplication of a vector by $\hat{A}^{L}$ (as required by one step of CG) in a logarithmic-linear complexity without building the entire matrix $\hat{A}^{L}$. 


\subsection{Multilevel FE space in D and preconditioning}

The first question to be addressed is therefore the choice of a basis in the sparse tensor space such that $\operatorname{cond}_{2}\left(\hat{A}^{L}\right)$ remains bounded as $L \rightarrow \infty$.

Assumption 1 There exists a family $\left\{\psi_{i}^{l}\right\}_{l \in \mathbb{N}_{0}, i \in \mathcal{I}^{l}} \subset H_{0}^{1}(D)$ such that each $u \in H_{0}^{1}(D)$ can be expanded as a convergent series in $H_{0}^{1}(D)$

$$
u=\sum_{l \in \mathbb{N}_{0}} \sum_{i \in \mathcal{I}^{l}} c_{i}^{l} \psi_{i}^{l}
$$

and the following 'stability condition' is fulfilled

$$
\sum_{l \in \mathbb{N}_{0}} \sum_{i \in \mathcal{I}^{l}}\left(c_{i}^{l}\right)^{2} \sim\left\|\sum_{l \in \mathbb{N}_{0}} \sum_{i \in \mathcal{I}^{l}} c_{i}^{l} \psi_{i}^{l}\right\|_{H_{0}^{1}(D)}^{2} .
$$

We present some examples of families satisfying Assumption 1.

Example 2 For $D=] 0,1$, let us consider $\phi$ the hat function on $\mathbb{R}$, piecewise linear, taking values $(0,1,0)$ at $(0,1 / 2,1)$ and vanishing outside $D$. We set $\mathcal{I}^{l}:=\left\{1,2, \ldots, 2^{l}\right\}$ and $\psi_{i}^{l}(x):=2^{-l / 2} \phi\left(2^{l} x-i+1\right), x \in D$. The family thus obtained satisfies then Assumption 1.

Example 3 With $D, \mathcal{I}^{l}$ and $\phi$ as above, we define on $\mathbb{R}$ the function $\psi$, piecewise linear, taking values $(0,1,-6,10,-6,1,0)$ in consecutive points $(0,1 / 2,1,3 / 2,2,5 / 2,3)$ and vanishing outside ]0,3[. Similarly, $\psi^{\star}$ take $(0,9,-6,1,0)$ at $(0,1 / 2,1,3 / 2,2)$ and $\psi_{\star}$ assumes values $(0,1,-6,9,0)$ at $(0,1 / 2,1,3 / 2,2)$. Further, we define $\psi_{1}^{0}:=\phi$ (scaling function) and $\psi_{1}^{l}(x):=2^{-l / 2} \psi^{\star}\left(2^{l} x\right), \psi_{2^{l}}^{l}:=2^{-l / 2} \psi_{\star}\left(2^{l} x-2^{l}+1\right), x \in D$, for $l \geq 1$ (boundary wavelets). Analogously, $\psi_{i}^{l}(x):=2^{-l / 2} \psi\left(2^{l} x-i+2\right), x \in D$ for $2 \leq i \leq 2^{l}-1$ and $l \geq 2$ (interior wavelets). The family thus obtained satisfies Assumption 1.

Remark 8 Higher order functions satisfying Assumption 1 exist as well (see, e.g. [3]). In the case $D$ is an arbitrary polygon, bases satisfying Assumption 1 can also be constructed. See [4] for examples.

Example 4 If $D=] 0,1{ }^{d}$, we consider the index set $\mathcal{I}^{l}:=\left\{(j, k) \in \mathbb{N}_{0}^{d} \times\right.$ $\left.\mathbb{N}_{0}^{d} \mid \max _{1 \leq q \leq d} j_{q}=l, 1 \leq k_{q} \leq 2^{j_{q}}\right\}$. Then, starting from the family in Example 3, we put $\psi_{i}^{l}(x)=\prod_{q=1}^{d} \psi_{k_{q}}^{j_{q}}\left(x_{q}\right) \quad \forall x=\left(x_{q}\right)_{1 \leq q \leq d} \in D$, with $i=(j, k)$, to obtain (after rescaling) a family which satisfies Assumption 1 (see [10]).

Returning to the family $\left\{\psi_{i}^{l}\right\}_{l \in \mathbb{N}_{0}, i \in \mathcal{I}^{l}}$ provided by Assumption 1, an increasing sequence of FE spaces in $D$ can be defined in terms of these functions by

$$
V^{L}:=\operatorname{Span}\left\{\psi_{i}^{l} \mid 0 \leq l \leq L, i \in \mathcal{I}^{l}\right\}
$$


An algebraic complement $W^{L}$ of $V^{L-1}$ in $V^{L}$ is explicitly given by

$$
W^{L}:=\operatorname{Span}\left\{\psi_{i}^{L} \mid i \in \mathcal{I}^{L}\right\}
$$

We define the index set

$$
\hat{\mathcal{I}}^{L}:=\bigcup_{l_{1}+l_{2}=L} \mathcal{I}^{l_{1}} \times \mathcal{I}^{l_{2}}
$$

as a direct union, and for $\mathbf{i}:=\left(i_{1}, i_{2}\right) \in \mathcal{I}^{l_{1}} \times \mathcal{I}^{l_{2}} \subset \hat{\mathcal{I}}^{l_{1}+l_{2}}$ we consider the functions

$$
\psi_{\mathbf{i}}^{l}:=\psi_{i_{1}}^{l_{1}} \otimes \psi_{i_{2}}^{l_{2}}, \quad \text { with } l:=l_{1}+l_{2} .
$$

Since they are linearly independent, we obtain, via (5.10), the following explicit description of the sparse tensor space $\hat{V}^{L}$ through a basis,

$$
\hat{V}^{L}=\operatorname{Span}\left\{\psi_{\mathbf{i}}^{l} \mid 0 \leq l \leq L, \quad \mathbf{i} \in \hat{\mathcal{I}}^{l}\right\}
$$

An algebraic excess $\hat{W}^{L}$ of the sparse tensor scale $\left\{\hat{V}^{L}\right\}_{L \geq 0}$ can be defined by

$$
\hat{W}^{L}:=\operatorname{Span}\left\{\psi_{\mathbf{i}}^{L} \mid \mathbf{i} \in \hat{\mathcal{I}}^{L}\right\}
$$

and this space can be further decomposed as

$$
\hat{W}^{L}=\bigoplus_{|\mathbf{l}|=L} W^{\mathbf{l}} \quad \text { with } \quad W^{\mathbf{l}}:=\operatorname{Span}\left\{\psi_{\mathbf{i}}^{|\mathbf{l}|} \mid \mathbf{i} \in \mathcal{I}^{l_{1}} \times \mathcal{I}^{l_{2}}\right\},
$$

where $|\mathbf{I}|:=l_{1}+l_{2}$ for $\mathbf{I}=\left(l_{1}, l_{2}\right) \in \mathbb{N}_{0}^{2}$.

For further reference, let us collect, for $L \geq 0$, in a column vector denoted $\Psi^{L}$, the basis functions in the definition (6.6) of $W^{L}$. Similarly, for $\mathbf{l} \in \mathbb{N}_{0}^{2}$ let $\Psi^{\mathbf{l}}$ be the column vector containing the basis functions of $W^{\mathbf{l}}$, as defined in (6.11). By nnz we denote the number of nonzero entries of a matrix.

Proposition 14 i) The matrix $\hat{A}^{L}$ has uniformly bounded condition number, as $L \rightarrow \infty$.

ii) The matrix $\hat{A}^{L}$ is not sparse, in the sense that $\mathrm{nnz}\left(\hat{A}^{L}\right) \geq O\left(\left(N^{L}\right)^{2}\right)$ (compare (5.19))

Proof. i) (6.4) means that

$$
u=\sum_{l \geq 0} \sum_{i \in \mathcal{I}^{l}} c_{i}^{l} \psi_{i}^{l} \longrightarrow|u|_{w}^{2}:=\sum_{l \geq 0} \sum_{i \in \mathcal{I}^{l}}\left(c_{i}^{l}\right)^{2}
$$

defines an equivalent norm on $H_{0}^{1}(D)$. The same holds then for the basis $\left\{\psi_{\mathbf{i}}^{l}\right\}_{l \in \mathbb{N}_{0}, \mathbf{i} \in \hat{\mathcal{I}}^{l}}$ introduced in (6.8) w.r.t. the space $H_{(0)}^{1,1}(D \times D)$. It follows that 
for $\underline{C}:=\left(C_{\mathbf{i}}^{l}\right)_{l, \mathbf{i}} \in \mathbb{R}^{\hat{N}^{L}}$ (recall that $\left.\hat{N}^{L}:=\operatorname{dim} \hat{V}^{L}\right), C:=\sum_{l=0}^{L} \sum_{\mathbf{i} \in \hat{\mathcal{I}}^{l}} C_{\mathbf{i}}^{l} \psi_{\mathbf{i}}^{l}$ is an element of $\hat{V}^{L}$ and

$$
\left\langle\hat{A}^{L} \underline{C}, \underline{C}\right\rangle_{\mathbb{R}^{\hat{N}^{L}}}=\mathcal{Q}(C, C) \sim\|C\|_{H_{(0)}^{1,1}(D \times D)}^{2} \sim \sum_{l=0}^{L} \sum_{\mathbf{i} \in \hat{\mathcal{I}}^{l}}\left(C_{\mathbf{i}}^{l}\right)^{2}=\|\underline{C}\|_{\mathbb{R}^{\hat{N}^{L}}}^{2}
$$

As for ii), one can easily see that for all the examples we considered before, the entries of $\hat{A}^{L}$ corresponding to the basis functions $\psi_{i_{1}}^{l_{1}} \otimes \psi_{i_{2}}^{0}$ and $\psi_{i_{3}}^{0} \otimes \psi_{i_{4}}^{l_{2}}$ for all admissible indices $j_{1}, j_{2}, i_{1}, i_{2}, i_{3}, i_{4}$ are in general nonzero, implying the desired lower bound.

\subsection{Matrix-vector multiplication}

The nonsparsity makes the storage and use of $\hat{A}^{L}$ rather costly. Taking into account the structure of $\hat{A}^{L}$, one should store only the matrix $A^{L}$ corresponding to the discrete mean field problem (5.4) and relate $\hat{A}^{L}$ to $A^{L}$ to perform one step of the CG algorithm.

Elementary considerations show that for every $C_{u}=\left(\underline{C}_{u}^{\mathbf{l}}\right)^{\top} \cdot \Psi^{\mathbf{l}} \in$ $\hat{V}^{L}, C=\left(\underline{C}^{\mathbf{l}}\right)^{\top} \cdot \Psi^{\mathbf{l}} \in \hat{V}^{L}$,

$$
\mathcal{Q}\left(C_{u}, C\right)=\sum_{l, l^{\prime}=0}^{L} \sum_{\substack{l,|=l\\| I^{\prime} \mid=l^{\prime}}}\left(\underline{C}_{u}^{\mathbf{l}}\right)^{\top} \cdot\left(A_{l_{1}, l_{1}^{\prime}}^{L} \otimes A_{l_{2}, l_{2}^{\prime}}^{L}\right) \underline{C}^{\mathbf{l}^{\prime}},
$$

where $A_{l, l^{\prime}}^{L}:=q\left(\Psi^{l}, \Psi^{l^{\prime}}\right), \forall 0 \leq l, l^{\prime} \leq L$ are the blocks of the stiffness matrix $A^{L}$ of the mean field problem (5.4) in $D$ describing the interactions between levels $l$ and $l^{\prime}$. This shows that the stiffness matrix $\hat{A}^{L}$ of the correlation problem computed w.r.t. the hierarchical basis (6.9) of $\hat{V}^{L}$ has a block structure and each block is a tensor product of certain blocks of the stiffness matrix $A^{L}$ of (5.4).

We assume $A^{L}$ to be sparse in the following sense.

Assumption 2 The number of nonzero entries of the block $A_{l, l^{\prime}}^{L}$ admits the upper bound

$$
\operatorname{nnz}\left(A_{l, l^{\prime}}^{L}\right) \lesssim\left(\min \left(l, l^{\prime}\right)+1\right)^{d-1} 2^{d \max \left\{l, l^{\prime}\right\}}, \quad \forall 0 \leq l, l^{\prime} \leq L,
$$

for some $d \in \mathbb{N}$ and with a constant depending only on $d$.

We remark that for each of the previously mentioned examples, as well as for similar wavelet constructions, Assumption 2 holds.

Under Assumption 2, and using (6.13), the following algorithm realizes the multiplication $\underline{C} \rightarrow \hat{A}^{L} \underline{C}$ in log-linear complexity. Here $\operatorname{Id}_{l, l}$ stands for the identity matrix of size $\operatorname{dim}\left(W^{l}\right)$. 
Algorithm 3 Assume (6.14).

store $A^{L}=\left(A_{l, l^{\prime}}^{L}\right)_{0 \leq l, l^{\prime} \leq L}$ and $x=\left(x_{1}\right)_{l_{1}+l_{2} \leq L}, O\left(L^{d} 2^{d L}\right)$ numbers

for $\mathbf{I}=\left(l_{1}, l_{2}\right)$ satisfying $l_{1}+l_{2} \leq L$

initialize $\left(\hat{A}^{L} x\right)_{\mathbf{l}}:=0$;

for $\mathbf{l}^{\prime}=\left(l_{1}^{\prime}, l_{2}^{\prime}\right)$ satisfying $l_{1}^{\prime}+l_{2}^{\prime} \leq L$

if $l_{1}+l_{2}^{\prime} \leq l_{1}^{\prime}+l_{2}$

else

$$
y_{\mathbf{l}}:=\left(\operatorname{Id}_{l_{1}, l_{1}} \otimes A_{l_{2}, l_{2}^{\prime}}^{L}\right)\left(\left(A_{l_{1}, l_{1}^{\prime}}^{L} \otimes \operatorname{Id}_{l_{2}^{\prime}, l_{2}^{\prime}}\right) x_{\mathbf{l}^{\prime}}\right)
$$

end $\%$ if

$$
y_{\mathbf{l}}:=\left(A_{l_{1}, l_{1}^{\prime}}^{L} \otimes \operatorname{Id}_{l_{2}, l_{2}}\right)\left(\left(\operatorname{Id}_{l_{1}^{\prime}, l_{1}^{\prime}} \otimes A_{l_{2}, l_{2}^{\prime}}^{L}\right) x_{\mathbf{I}^{\prime}}\right) ;
$$

update $\left(\hat{A}^{L} x\right)_{\mathbf{l}}:=\left(\hat{A}^{L} x\right)_{\mathbf{l}}+y_{\mathbf{l}}$;

end $\%$ for

end $\%$ for

We emphasize that the order in the block multiplication of Algorithm 3 is essential for the following bound on its complexity.

\subsection{Complexity}

Theorem 4 Under Assumption 2, Algorithm 3 performs the matrix-vector multiplication $x \rightarrow \hat{A}^{L} x$ in $O\left(\left(\log N^{L}\right)^{2 d+2} N^{L}\right)$ floating point operations. Besides, it requires only storage of $x$ and of the stiffness matrix $A^{L}$ of the mean field problem (5.4) in a hierarchical basis, that is $O\left(L^{d} 2^{d L}\right)$ memory.

Proof. Due to (6.13), we can write

$$
\left(\hat{A}^{L} x\right)_{\mathbf{I}}=\sum_{\left|\mathbf{I}^{\prime}\right| \leq L}\left(A_{l_{1}, l_{1}^{\prime}}^{L} \otimes A_{l_{2}, l_{2}^{\prime}}^{L}\right) \cdot x_{\mathbf{I}^{\prime}} .
$$

To perform the multiplication under the summation above, we note that

$$
\begin{aligned}
A_{l_{1}, l_{1}^{\prime}}^{L} \otimes A_{l_{2}, l_{2}^{\prime}}^{L} & =\left(A_{l_{1}, l_{1}^{\prime}}^{L} \otimes \operatorname{Id}_{l_{2}, l_{2}}\right) \cdot\left(\operatorname{Id}_{l_{1}^{\prime}, l_{1}^{\prime}} \otimes A_{l_{2}, l_{2}^{\prime}}^{L}\right) \\
& =\left(\operatorname{Id}_{l_{1}, l_{1}} \otimes A_{l_{2}, l_{2}^{\prime}}^{L}\right) \cdot\left(A_{l_{1}, l_{1}^{\prime}}^{L} \otimes \operatorname{Id}_{l_{2}^{\prime}, l_{2}^{\prime}}\right) .
\end{aligned}
$$

To multiply the 1.h.s. of (6.16) by $x_{\mathbf{I}^{\prime}}$, we have two possibilities: we can use either the r.h.s. of (6.16), or the r.h.s. of (6.17) (explicit building of the tensor block has been already ruled out, cf. Proposition 14 ii.). We choose the cheapest of the two, in terms of floating point operations, and that is what Algorithm 3 actually does through its selection statement.

Suppose therefore that $l_{1}+l_{2}^{\prime} \leq l_{1}^{\prime}+l_{2}$, so that we choose (6.17) over (6.16), as indicated in the algorithm. On account of (6.14), we deduce that 
the number $\#_{1}$ of floating point operations required by the first multiplication in the computation of $y_{1}$ can be estimated by

$$
\#_{1} \leq \operatorname{nnz}\left(A_{l_{1}, l_{1}^{\prime}}^{L} \otimes \operatorname{Id}_{l_{2}^{\prime}, l_{2}^{\prime}}\right) \lesssim\left(\min \left(l_{1}, l_{1}^{\prime}\right)+1\right)^{d-1}\left(l_{2}^{\prime}+1\right)^{d} 2^{d\left(\max \left\{l_{1}, l_{1}^{\prime}\right\}+l_{2}^{\prime}\right)} .
$$

Using the same argument, the subsequent multiplication requires an additional amount of floating point operations, $\#_{2}$, for which it holds

$$
\#_{2} \leq \operatorname{nnz}\left(\operatorname{Id}_{l_{1}, l_{1}} \otimes A_{l_{2}, l_{2}^{\prime}}^{L}\right) \lesssim\left(\min \left(l_{2}, l_{2}^{\prime}\right)+1\right)^{d-1}\left(l_{1}+1\right)^{d} 2^{d\left(\max \left\{l_{2}, l_{2}^{\prime}\right\}+l_{1}\right)} .
$$

We note that $l_{1}+l_{2}^{\prime} \leq l_{1}^{\prime}+l_{2}$ implies

$$
\max \left\{l_{1}, l_{1}^{\prime}\right\}+l_{2}^{\prime}, \max \left\{l_{2}, l_{2}^{\prime}\right\}+l_{1} \leq \max \left\{l_{1}+l_{2}, l_{1}^{\prime}+l_{2}^{\prime}\right\}
$$

It is easily seen then, using (6.18), that the multiplication under the summation in (6.15) can be performed using (for $L \geq 1$ )

$$
\#_{1}+\#_{2} \lesssim\left(\max \left\{l_{1}+l_{2}, l_{1}^{\prime}+l_{2}^{\prime}\right\}\right)^{2 d-1} 2^{d \max \left\{l_{1}+l_{2}, l_{1}^{\prime}+l_{2}^{\prime}\right\}}
$$

flops, with a constant which depends only on $d$.

Note that in the case $l_{1}+l_{2}^{\prime}>l_{1}^{\prime}+l_{2}$, one should use (6.16) to perform the multiplication in (6.15). Symetrically, the computational cost admits also in this case the upper bound (6.19). From (6.15) and (6.19) follows that the number of operations needed to perform $x \rightarrow \hat{A}^{L} x$ (collect all blocks $\left(\hat{A}^{L} x\right)_{1}$ for $\mathbf{I}=\left(l_{1}, l_{2}\right) \in \mathbb{N}_{0}^{2}$ subject to $\left.|\mathbf{l}|=l_{1}+l_{2} \leq L\right)$ is bounded from above by

$$
\sum_{l_{1}+l_{2} \leq L} \sum_{l_{1}^{\prime}+l_{2}^{\prime} \leq L}\left(\max \left\{l_{1}+l_{2}, l_{1}^{\prime}+l_{2}^{\prime}\right\}\right)^{2 d-1} 2^{d \max \left\{l_{1}+l_{2}, l_{1}^{\prime}+l_{2}^{\prime}\right\}} \text {. }
$$

Since for a given $l \geq 0$ the equation $l_{1}+l_{2}=l$ has exactly $l+1$ solutions $\left(l_{1}, l_{2}\right) \in \mathbb{N}_{0}^{2}$, we conclude that the total number of flops \# required by the multiplication $x \rightarrow \hat{A}^{L} x$ satisfies

$$
\# \lesssim \sum_{l=0}^{L} l^{3} l^{2 d-1} 2^{d l}=O\left(L^{2 d+2} 2^{d L}\right)=O\left(\left(\log N^{L}\right)^{2 d+2} N^{L}\right) .
$$

Since the number of steps required by the CG algorithm to compute the discrete solution up to a prescribed accuracy is bounded provided that the stiffness matrix is well-conditioned (ensured here via Proposition 14) and that we use the FE solution at level $L-1$ as initial guess for the level $L$, we obtain

Theorem 5 The deterministic problem (3.4) for the correlation function $C_{u} \in H^{s+1, s+1}(D \times D) \cap H_{(0)}^{1,1}(D \times D)$ of the random solution $u$ to $(2.5)$ is numerically solvable at a cost of

$$
O\left(\left(\log N^{L}\right)^{2 d+2} N^{L}\right)
$$


floating point operations, with

$$
O\left(\left(\log N^{L}\right)^{d} N^{L}\right)
$$

memory, for a relative accuracy in $H^{1,1}(D \times D)$ of

$$
O\left(\left(\log N^{L}\right)^{1 / 2}\left(N^{L}\right)^{-\delta}\right),
$$

where $\delta=\min \{p, s\} / d$ and $N^{L}$ denotes the number of degrees of freedom for the mean field problem.

Up to logarithmic terms, the estimates (6.20), (6.21), (6.22) are similar to those of the mean field problem (3.2).

Remark 9 If the domain $D \subset \mathbb{R}^{d}$ has itself a product structure, for instance $D=D_{1} \times D_{2}$ or $\left.D=\right]-a, a\left[^{d}\right.$, sparse grids w.r.t. this product structure can be used to further reduce the complexity of the correlation problem (3.4) (see e.g. [11]). Note, however, that this requires regularity of the correlation kernel $C_{u}$ on the corresponding anisotropic Sobolev scale in $D$, which can in turn be ensured only by a much higher, even unrealistic regularity of $C_{u}$ (or of $u(\cdot, \omega)$ ) on the scale naturally associated to the Laplace operator acting in $D$, the isotropic Sobolev scale.

\section{Numerical experiments}

We present here elementary numerical results that are to be compared with the theoretical ones we have obtained in Sections 5 and 6. We include the two examples introduced in Section 4, involving exponential and Dirac correlation (for simplicity we assume that $D=(-1,1)$ and $A=1$ ). We investigate then a third situation in which the coefficient $A$ is non-constant. We mention that each figure presents two curves: the one corresponding to the theoretical result (dashed) and the one obtained numerically (solid). We also mention that in all these cases, the hat-function basis from Example 2 has been used to perform numerical algorithms.

The first example to be considered is therefore the Dirichlet problem

$$
\left\{\begin{aligned}
L\left(\partial_{x}\right) L\left(\partial_{y}\right) C_{u} & =e^{-|x-y|} & & \text { in } L^{2}\left((-1,1)^{2}\right) \\
\gamma_{0}\left(C_{u}\right) & =0 & & \text { on } \partial(-1,1)^{2},
\end{aligned}\right.
$$

with $A(x)=\operatorname{Id}_{\mathbb{R}}, \forall x \in D=(-1,1)$, that is, $L\left(\partial_{x}\right)=-\Delta_{x}$.

Figure 1 shows the convergence of the FE solution in this simple case, with non-singular (but also non-smooth) data and constant coefficient $A$. From Theorem 5 follows that for this particular choice of data the rate of convergence equals $(\log N)^{3 / 2} N^{-1}$, where throughout this section $N$ stands for the number of degrees of freedom. 


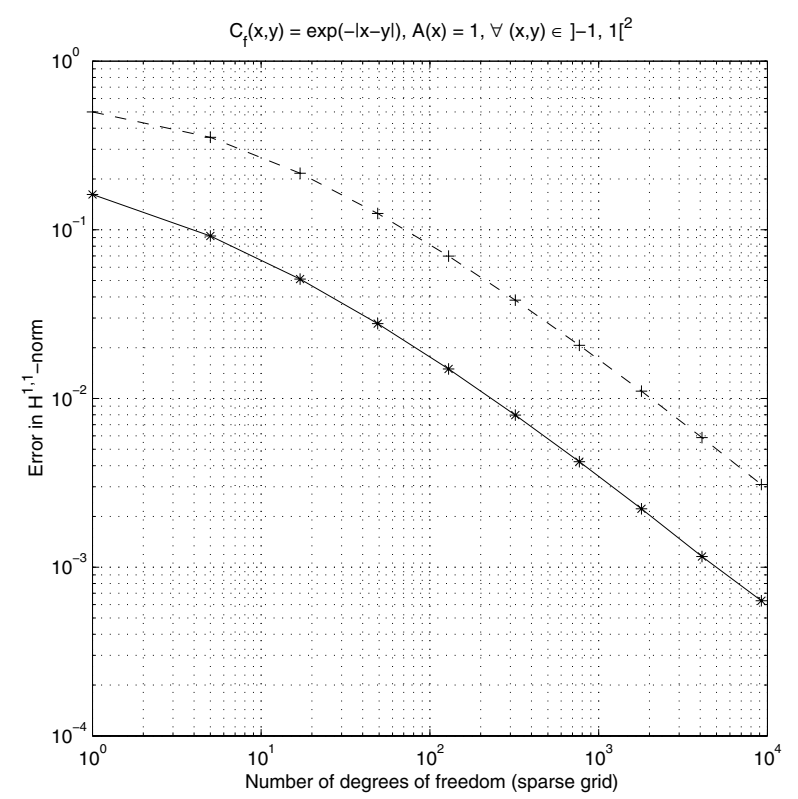

Fig. 1. Convergence in the case of exponential r.h.s. and constant coefficient $A=1$ (solid) and the bound (6.22), with $\delta=1$ (dashed)

Our next example is the one-dimensional white noise (see (4.12)), with the singular r.h.s. $C_{f}=\delta(x-y)$. Thus the problem reads

$$
\left\{\begin{aligned}
L\left(\partial_{x}\right) L\left(\partial_{y}\right) C_{u} & =\delta(x-y) & & \text { in the dual space of } H_{0}^{1,1}\left((-1,1)^{2}\right) \\
\gamma_{0}\left(C_{u}\right) & =0 & & \text { on } \partial(-1,1)^{2}
\end{aligned}\right.
$$

where $L\left(\partial_{x}\right)=-\Delta_{x}$. Figure 2 shows the convergence of the FE-solution. The theoretical convergence rate is here $(\log N)^{5 / 4} N^{-3 / 4}$, again as a consequence of Theorem 5.

We conclude this section with a new example, in which all data are again smooth but the coefficient $A$ is no longer constant. More precisely, we choose the coefficent $A$ and the solution $C_{u}$ as follows:

$$
A(x)=2+\sin (\pi x), \quad C_{u}(x, y)=\left(1-x^{2}\right)\left(1-y^{2}\right) e^{x y}
$$

for all $x, y \in D=(-1,1)$. The numerical results are shown in Figure 3. As in the first example, the error decays as $(\log N)^{3 / 2} N^{-1}$ when $N \rightarrow \infty$. This curve, as well as those we have plotted before, does not have the appearance of a straight line and this is due to the logarithmic terms arising in the error estimates by sparse grids. Of course, asymptotically as $N \rightarrow \infty$, these terms do not play an essential role, but it turns out that their influence is rather strong, within the computational range. 


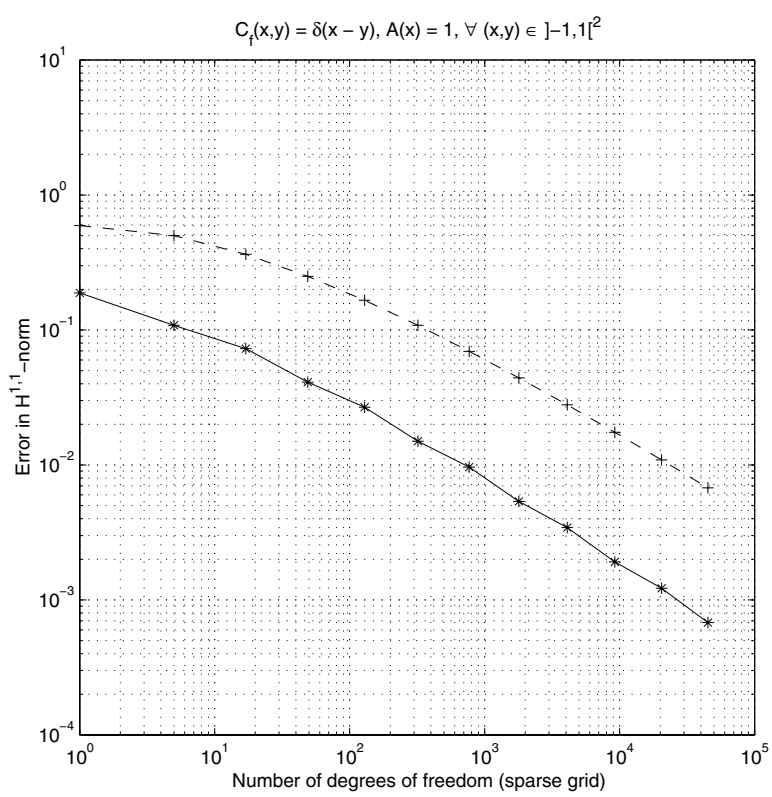

Fig. 2. Convergence in the case of singular r.h.s. and constant coefficient $A=1$ (solid) and the bound (6.22), with $\delta=3 / 4$ (dashed)

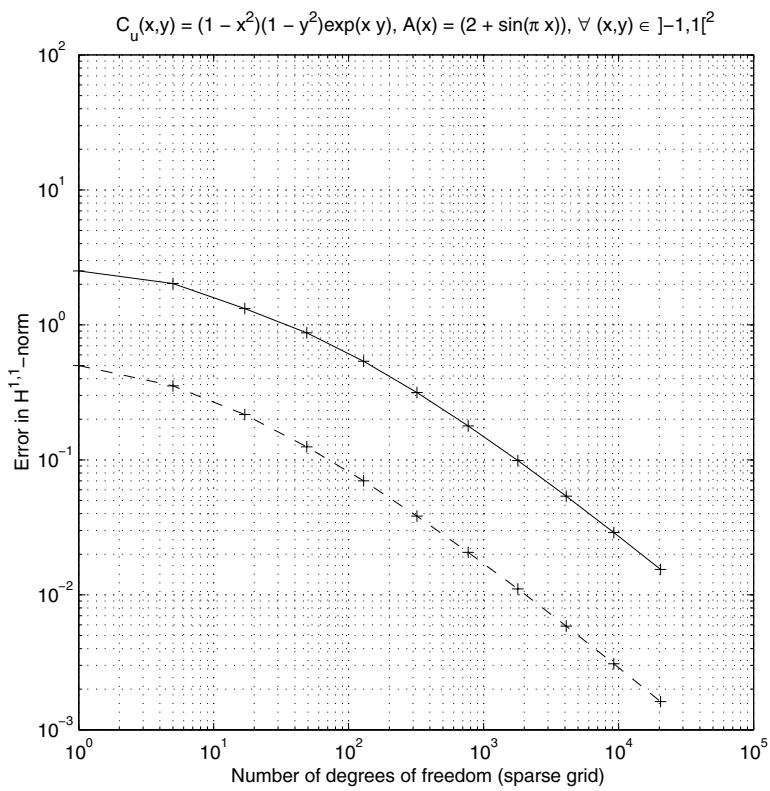

Fig. 3. Convergence in the case of non-constant coefficient $A$ (solid) and the bound (6.22), again with $\delta=1$ (dashed) 


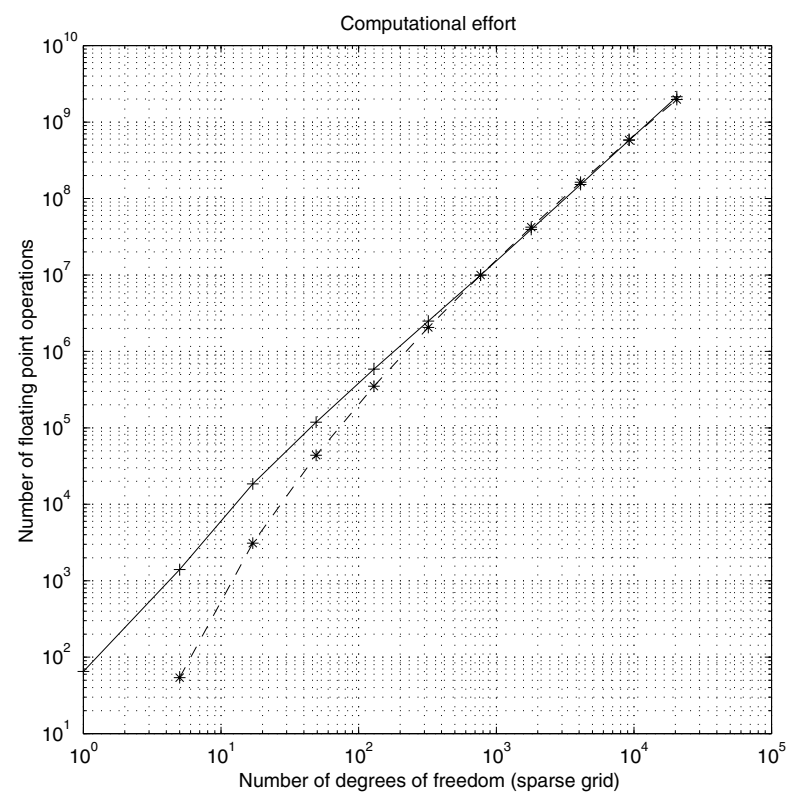

Fig. 4. Comparison between the effort required by the standard CG method based on Algorithm 3 (solid) and its theoretical estimate given in Theorem 5 by (6.20) (dashed)

Finally, Figure 4 shows the performance of Algorithm 3 matching the theoretical estimate concerning the computational effort given by (6.20), namely \#flops $\sim\left(\log N^{L}\right)^{5} \cdot N^{L}$. We mention that this analysis has been done for the same example (7.1), and that the solution at each level $L$ has been computed directly, without using the solution at level $L-1$ as initial guess.

\section{References}

[1] Babuška, I.: On Randomised Solutions of Laplace's Equation. Časopis pro Pěstováni Matematiky 86, 269-275 (1961)

[2] Babuška, I., Guo, B.Q.: Regularity of the solution of elliptic problems with piecewise analytic data. Part I. Boundary value problems for linear elliptic equation of second order. SIAM J. Math. Anal. 19, 172-203 (1988)

[3] Dahmen, W., Kunoth, A., Urban, K.: Biorthogonal Spline-Wavelets on the Interval - Stability and Moment Conditions. Appl. Comp. Harm. Anal. 6, 132-196 (1999)

[4] Dahmen, W., Stevenson, R.: Element-by-Element Construction of Wavelets Satisfying Stability and Moment Conditions. SIAM J. Numer. Anal. 37, 319-352 (1999)

[5] Deb, M.K., Babuška, I., Oden, J.T.: Solution of Stochastic Partial Differential Equations Using Galerkin Finite Element Techniques. Preprint, University of Texas at Austin, 2001

[6] Dettinger, M.: Numerical Modeling of Aquifer Systems Under Uncertainty: A Second Moment Analysis, MSc. Thesis, MIT, Department of Civil Engineering, 1979 
[7] Ghanem, R.G., Spanos, P.D.: Stochastic finite elements: a spectral approach, Springer-Verlag, 1991

[8] Gilbarg, D., Trudinger, N.S.: Elliptic Partial Differential Equations of Second Order, Springer-Verlag, 1977

[9] Golub, G., Van Loan, C.F.: Matrix Computations, 4th edition, Johns Hopkins University Press, 1996

[10] Griebel, M., Oswald, P.: Tensor product type subspace splittings and multilevel iterative methods for anisotropic problems. Adv. Comput. Math. 4, 171-206 (1999)

[11] Griebel, M., Oswald, P., Schiekofer, T.: Sparse grids for boundary integral equations. Numer. Mathematik 83, 279-312 (1999)

[12] Holden, H., Oksendal, B., Uboe, J., Zhang, T.: Stochastic Partial Differential Equations: A Modeling, White Noise Functional Approach. Birkhäuser, 1996

[13] Kampé de Fériet, J.: Random Solutions of Partial Differential Equations, Proc. of the Third Berkeley Symp. on Math. Statistics and Probability, III 199-208

[14] Keller, J.B.: Stochastic Equations and Wave Propagation in Random Media, Bellman, 1964

[15] Kleiber, M., Hien, T.D.: The Stochastic Finite Element Method. John Wiley \& Sons, 1992

[16] Kloeden, P.E., Platen, E.: Numerical solution of stochastic differential equations. 3rd edition, Springer-Verlag, 1999

[17] Matache, A.M.: Sparse Two Scale FEM for Homogenization Problems. J. Sc. Comp. 17, 659-669 (2002)

[18] Oksendal, B.: Stochastic differential equations: an introduction with applications, 3rd edition. Springer-Verlag, 1992

[19] Protter, P.: Stochastic integration and differential equations: a new approach, 3rd edition, Springer-Verlag, 1995

[20] Strichartz, R.S.: A guide to distribution theory and Fourier transforms, CRC Press, Boca Raton, 1994

[21] Todor, R.A.: Doctoral Dissertation ETHZ. In preparation

[22] Yaglom, A.M.: An Introduction to the Theory of Stationary Random Functions. Prentice-Hall, Englewood Cliffs, New Jersey, 1962

[23] Yosida, K.: Functional Analysis, Springer-Verlag, 1964

[24] Zenger, Ch.: Sparse Grids, Parallel Algorithms for PDE's-Proceedings of the 6th GAMM-Seminar, Kiel, 1990 